\title{
KILLED ANTI-TUBERCULOSIS VACCINE AND HYALURONIDASE (DIFFUSING VACCINE)
}

\author{
BY
}

\author{
G. SALVIOLI \\ From the Institute of Paediatrics, Bologna, Italy
}

(RECEIVED FOR PUBLICATION OCTOBER 27, 1952)

Hyaluronidase was first used in human antituberculosis vaccination in 1950 (Salvioli, 1933).

From the very first it was possible to see that the addition of hyaluronidase increases remarkably the vaccinating activity of killed tubercle bacilli injected intradermally. Also it was confirmed that their diffusion offered an interesting field of study not only of vaccinating antigens consisting of microbic bodies or of organized structures, but also of extractive antigens, of immune sera and of antibiotic drugs (Salvioli, 1933).

The hypothesis that led to the use of a vaccine associated with hyaluronidase, termed diffusing vaccine (V.D.S.*), in anti-tuberculosis vaccination, was the same that about 20 years ago led me to perform intradermal vaccinations with Anatubercolina $I$. petragnani, using a plurifocal method (V.D.S.). The aim of this latter method is to produce a vast tissue process with an adequate dose of vaccine.

Anti-tuberculosis vaccination with killed bacilli with the addition of diffusing vaccine aims at vaccinating and protecting the organism by using only one injection of a reduced amount of vaccine. The focus of injection often appears less prominent than with the use of germs without hyaluronidase; it is more extensive, showing that the region of tissue involved in the constitution of the vaccinial focus is greater. As I pointed out many years ago, the vaccinial focus is the essential factor for the creation of resistance against virulent tubercle bacilli produced by a vaccine.

Killed tubercle bacilli have been used in V.D.S. vaccination tests for two years. Their vaccinating and protective properties were widely shown in practice in newborn children (Salvioli, 1951a and b) as well as in various experiments in guinea-pigs and dogs.

In man V.D.S. vaccination has been predominantly employed in infants, but some older individuals who had no allergy have also been injected. The vaccine was inoculated intradermally into the palmar surface of the forearm and caused no general disturbances.

\footnotetext{
* Known in Italy as V.D.S. (vaccino diffondente Salvioli).
}

The vaccinial focus had a slower and much longer evolution than that following vaccination without the addition of hyaluronidase. As soon as allergy has developed there is a phenomenon of recidivation which may lead to the formation of small superficial eschars and moderate secretion, especially in older individuals. The scar is mostly smooth or linear, and may even be absent.

Allergy appears after three or four months (Fig. 1).

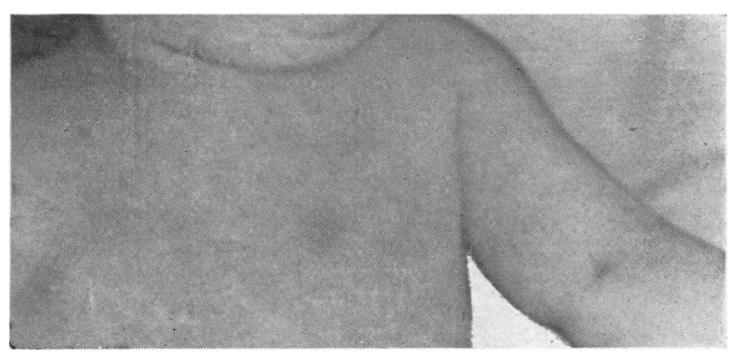

Fig. 1.-Vaccinial focus on the forearm five months after vaccination positive plaster-reaction on the chest.

In some subjects in whom the vaccinial process is very slow allergy may appear as late as six months after vaccination, but this is an exceptional occurrence. The percentage of allergy after one year is about 95. These results were obtained with relatively moderate doses compared with other vaccination methods; the doses were further reduced according to the age of the patient.

An explanation of the better action of vaccine associated with hyaluronidase is provided by studies made on rabbits in the Anti-tuberculosis Vaccinioprophylactic Centre of Bologna (Salvioli, Degli Esposti and Dina, 1952a and b). Histochemical tissue reactions produced by vaccines of heat-killed tubercle bacilli with the addition of hyaluronidase were studied in comparison with those caused by the vaccine alone. It was found that the diffusion of killed tubercle bacilli causes a much greater inoculation focus in the skin than with tubercle bacilli 
alone; this focus shows slower development and less probability of eliminating the inoculated bacterial bodies.

Histochemically the results obtained with V.D.S. are the following. In the vaccination focus the typical specific tissue with granulomas of epitheloid giant cells and necrobiotic aspects are found. In the satellite (crural) lymph nodes there is in $60 \%$ of the cases a typical tuberculous reaction similar to that in the vaccinial focus. In the inguinal satellite nodes at the vaccinial focus tuberculous granulomas are always present together with a remarkable increase of the light-coloured centres, with numerous plasma cells, Russell's hyaline bodies and a diffuse proliferative histiocytic reaction. In the lymph nodes contralateral to the vaccinial focus the picture is very similar to the preceding one.

In the spleen an increased number of follicles and the formation of germinal centres are observed; in the pulp a proliferative hystiocytic reaction and numerous small foci showing haemopoietic activity are seen. Hyperaemia is marked and in the sinuses some large endothelial cells with phagocytic properties are present.

A swelling of Kupffer's cells is always present in the liver and sometimes, though more rarely, signs of mobilization.

The histochemical features encountered in individuals vaccinated with killed germs alone, except for the constant absence of specific lesions in the satellite lymph nodes at the vaccinial focus, are of the same type as those seen in rabbits vaccinated with V.D.S., but of lesser degree and constancy, especially in the findings of the spleen and liver. Pyroninophilia is much intenser in V.D.S.-vaccinated individuals, especially near the vaccination focus. Sivori's enzyme reaction gives almost double values in V.D.S. vaccination subjects, in comparison with those obtained by simple vaccination.

All these facts show that the diffusing factor produces in the killed germs that migratory property characteristic of living germs, thus determining a 'vaccination complex' analogous to Ranke's primary complex which is known to modify the reaction of the organism to subsequent contacts with tubercle bacilli.

The specific lesions of the satellite lymph nodes during diffusing vaccination represent a direct stimulus for a tissue containing many mesenchymal elements, thus greatly increasing the specific involvement of the reticulo-endothelial system.

The original type of diffusing vaccination with killed germs, therefore, produces the same characteristics as those obtained with living germs without, however, having their disadvantages.

The foregoing facts allow us to speculate on the mechanism of anti-tuberculosis and other vaccination, and on the constitution of various immunization phenomena, light on which may be obtained through a controlled use of the diffusing factor.

\section{REFERENCES}

Salvioli, G. (1933). Studi Fac. med. senese, 1, face. 2 (1950). Clin. pediat., Bologna, 32, 703.

(1951a). Boil. Sci. med., 123, 235.

(1951b). Atti X Congr. ital. Tisiologia, Napoli, vol. 1, p. 191. Degli Esposti, A. and Dina, M. A. (1952a). Clin. pediat., Bologna. 34. 137.

Boll. Ist. Sieroter, milan., 31. 209. 